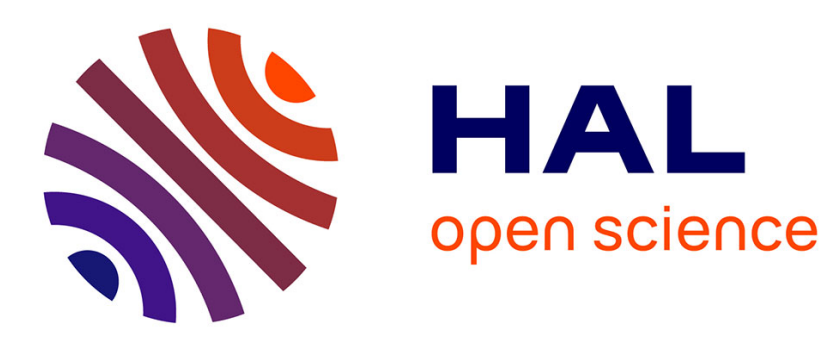

\title{
Scaling Theory of Two-Phase Dendritic Growth in Undercooled Ternary Melts
}

Silvère Akamatsu, Sabine Bottin-Rousseau, Gabriel Faivre, Efim A Brener

\section{To cite this version:}

Silvère Akamatsu, Sabine Bottin-Rousseau, Gabriel Faivre, Efim A Brener. Scaling Theory of Two-Phase Dendritic Growth in Undercooled Ternary Melts. Physical Review Letters, 2014, 112, pp.105502. 10.1103/PhysRevLett.112.105502 . hal-01472466

\section{HAL Id: hal-01472466 https://hal.science/hal-01472466}

Submitted on 20 Feb 2017

HAL is a multi-disciplinary open access archive for the deposit and dissemination of scientific research documents, whether they are published or not. The documents may come from teaching and research institutions in France or abroad, or from public or private research centers.
L'archive ouverte pluridisciplinaire HAL, est destinée au dépôt et à la diffusion de documents scientifiques de niveau recherche, publiés ou non, émanant des établissements d'enseignement et de recherche français ou étrangers, des laboratoires publics ou privés. 


\title{
Scaling theory of two-phase dendritic growth in undercooled ternary melts
}

\author{
Silvère Akamatsu, ${ }^{1,}$ * Sabine Bottin-Rousseau, ${ }^{1}$ Gabriel Faivre, ${ }^{1}$ and Efim A. Brener ${ }^{2}$ \\ ${ }^{1}$ Institut des Nanosciences de Paris, UMR 7588 CNRS, UPMC, 75005 Paris, France \\ ${ }^{2}$ Peter Grünberg Institut, Forschungszentrum Jülich, D-52425 Jülich, Germany
}

(Dated: February 6, 2014)

\begin{abstract}
Two-phase dendrites are needle-like crystals with a eutectic internal structure growing during solidification of ternary alloys. We present a scaling theory of these objects based on Ivantsov's theory of dendritic growth and Jackson and Hunt's theory of eutectic growth. The additional introduction of the relationship $\rho \sim \lambda$ ( $\rho$ : dendrite tip radius; $\lambda$ : eutectic interphase spacing) suggested by recent experimental results [Akamatsu et al., Phys. Rev. Lett., 104, 056101 (2010)] leads to a complete solution of the selection problem and to the scaling rule $\rho \sim v^{-1 / 2}$ ( $v$ : dendrite tip growth rate).
\end{abstract}

PACS numbers: 47.54.+r, 61.72.Mm, 81.10.Aj, 81.30.Fb

The term of two-phase dendrite is used in materials sciences to designate needle-shaped crystals with a fine two-phase internal structure like those observed during univariant solidification of three-component alloys $[1,2]$. Few detailed studies have so far been devoted to these objects. The first theoretical questions that have to be dealt with are whether two-phase dendrites can grow in a stationary way and, if so, whether anisotropy effects are crucial in the process. An answer to these questions has recently been provided by the finding of stationary spiral two-phase dendrites (for short, $s p$ dendrite) during directional solidification of a transparent nonfaceted ternary eutectic $[3,4]$. The properties of the $s p$ dendrites are illustrated in Fig. 1. Like one-phase dendrites, they can be subdivided into a tip region characterized by a smooth outer shape, and a tail region where this shape is disrupted by morphological instabilities. In the tip region, the outer shape is a paraboloid. The two-phase substructure is generated by a spiral eutectic pattern located at the tip and rotating at a constant rotation speed. Except close to the tip, an interphase spacing $\lambda$, which is approximately equal to the helix pitch, can be defined. More details are given below. For now, we simply stress the fact that the spiral mechanism makes possible a perfectly steady two-phase growth along the curved solid-liquid interface, and that this mechanism in no way involves anisotropy effects. This has recently been confirmed by phase-field numerical simulations of $s p$ dendrites performed in a model ternary system in which the level of interfacial anisotropy could be varied [5].

In this article, we present a scaling theory of the steady state of two-phase dendrites in free growth in a ternary system without surface tension anisotropy. Our main objective is to cast light on the mechanism of selection and the range of existence of this growth structure in terms of morphological and thermodynamical parameters. The paraboloid outer shape and the regular eutectic substructure of $s p$ dendrites suggest to view the diffusion field

*Electronic address: akamatsu@insp.jussieu.fr
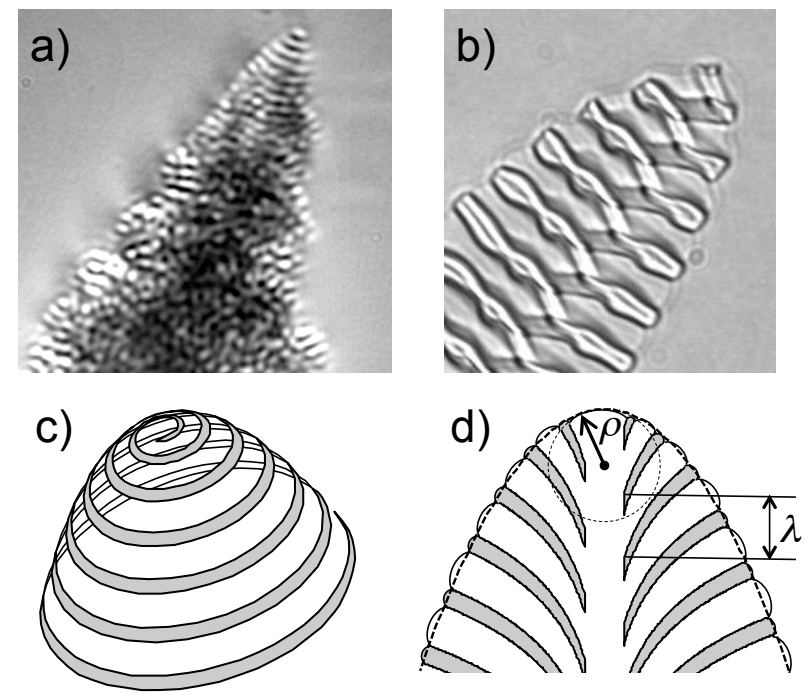

FIG. 1: Spiral two-phase dendrites. a and b: In situ optical micrographs taken during directional solidfication of a transparent ternary alloy (for experimental details, see [3]). a) bulk sample. Horizontal dimension: $105 \mu \mathrm{m}$. b) Thin samples. Horizontal dimension: $65 \mu \mathrm{m}$. c: Schematic 3D representation of the solid-liquid interface. One of the solid phase is grey, the other white. (d) Longitudinal section of the internal microstructure (dotted line: parabolic envelope).

outside two-phase dendrites as being composed of two parts: a long-range field, which only depends on the average composition of the liquid along the envelope of the dendrite, and obeys a theory similar to that of one-phase dendrites; a short-range field, which is driven by the differences in concentration in the liquid in front of the two eutectic phases, and is relevant to a theory similar to that of lamellar eutectic growth. We begin with a brief summary of these theories.

Consider the free growth of a one-phase dendrite in a AX binary alloy, where $\mathrm{X}$ is the solute. As a first step, the theory asumes that surface-tension effects are negligible. This implies that the solid-liquid interface is an isoconcentration surface, and that the solute mole fractions 
(for short, concentrations) of the liquid and the solid are those of the liquidus and solidus at the temperature $T$ of the system. Let $x$ and $x_{s}$ be these concentrations, respectively, and $x_{\infty}$ be the solute concentration far from the dendrite. Let $v$ be the growth speed of the dendrite tip, $\rho$ the tip radius, $D_{x}$ the diffusion coefficient of $\mathrm{X}$ in the liquid, and

$$
\Delta_{x}=\frac{x-x_{\infty}}{x-x_{s}}
$$

the degree of supersaturation of the liquid. Ivantsov demonstrated that all the paraboloids such that

$$
\frac{v \rho}{D_{x}}=F^{I v}\left(\Delta_{x}\right)
$$

where $F^{I v}\left(\Delta_{x}\right) \sim \Delta_{x} / \ln \left(\Delta_{x}^{-1}\right)$ when $\Delta_{x}<<1$ (which is the case we consider here), are steady-state solutions of the equations of growth without surface-tension effects [6]. In the next step an additional equation of the form $\rho^{2} v=$ constant is provided by introducing slightly anisotropic surface-tension or kinetic effects. As a result, $v$ and $\rho$ are uniquely determined and linked by a $\rho \sim v^{-1 / 2}$ scaling relationship at given $\Delta_{x}$. This has been established for both 2D [7-10] and 3D dendrite growth $[11,12]$. Similar selection and scaling rules for $v$ and $\rho$ have been found for various other needle-like crystal growth shape, such as the doublon (also called double finger) $[13,14]$ and the two-phase finger $[15,16]$. Unlike the dendrite, these last growth shapes are not crucially dependent on interfacial anisotropy and are not selected in orientation. On the other hand, like the dendrite, their shape does not depart much from an Ivantsov paraboloid, except at the tip, where a specific local structure ensures their stabilization and speed selection.

Consider now a AB binary alloy, where B is the solute, having a eutectic plateau at some temperature $T_{E}$ and a global concentration $c_{\infty}$ falling well inside this plateau. The growing solid contains two different crystal phases, namely, a A-rich $\alpha$ phase and a B-rich $\beta$ phase. At small solidification rates the system admits steady states consisting of a planar $\alpha \beta$ growth front with a uniform spacing $\lambda$ and a small average undercooling with respect to $T_{E}$. We summarize the Zener-Hillert-Jackson-Hunt (JH) theory of these states [17]. This theory was formulated for directional solidification in the $G / V \rightarrow 0$ limit and thus also holds in free growth. It is valid under the condition that $\lambda V / D<<1$, where $D$ is the diffusion coefficient of $\mathrm{B}$ in the liquid, which was verified during the experimental observation of $s p$ dendrites. The average concentration $c^{\alpha}$ of the liquid in front of the $\alpha$-liquid interfaces is larger than the average concentration $c^{\beta}$ in front of the $\beta$-liquid ones at the temperature $T$ of the growth front. This forms the basis of the exchange of solute between solid phases during growth. To a good approximation, $c^{\alpha}$ is on the $\alpha$ liquidus of the alloy and therefore is in equilibrium with a point $c_{s}^{\alpha}$ of the $\alpha$ solidus. The same applies to $c^{\beta}$ mutatis mutandis. JH showed that the mass conservation equation at the interface leads to

$$
P \frac{\lambda V}{D}=\Delta^{s o l}
$$

where

$$
\Delta^{s o l}=\frac{c^{\alpha}-c^{\beta}}{c_{s}^{\beta}-c_{s}^{\alpha}}
$$

and $P$ is a small $(<0.1)$ numerical factor occurring from the summation over the periodic structure. Note, incidentally, that the fraction $\eta$ of $\beta$ phase in the solid is related to $c_{\infty}$ by the global mass conservation equation $c_{\infty}=\eta c_{s}^{\beta}+(1-\eta) c_{s}^{\alpha}$. Furthermore, capillary effects (namely, the interface equilibrium conditions at the $\alpha$ - $\beta$ liquid junctions) generate a mean curvature of the solidliquid interface. This gives an additional "capillary" contribution to the average undercooling of the growth front, which reads

$$
\frac{d_{0}}{\lambda}=\Delta^{c a p}
$$

where $d_{0}$ is a material-dependent capillary length. The spacing value $\lambda_{J H}=\sqrt{P^{-1} d_{0} D V^{-1}}$ at which

$$
\Delta^{\text {sol }}=\Delta^{c a p}=\frac{\Delta_{E}}{2}
$$

is an important characteristic length for eutectic growth patterns. To be sure, there is no "strong selection" (in the sense that $\lambda$ tends towards $\lambda_{J H}$, or a value close to $\lambda_{J H}$, over time at constant $V$ ) of the spacing in binary eutectic growth, contrary to what was long believed. However, it is also true that any local structure containing $\lambda$ values deviating from $\lambda_{J H}$ by a factor of more than about 2 is short-lived so that, broadly speaking, $\lambda$ is almost always close to $\lambda_{J H}$, a fact which is sometimes referred to as "weak selection" [18-20].

Consider finally the directional solidification of a $\alpha \beta$ solid in a ABX ternary alloy, as studied in Ref. [3]. The rejection of $\mathrm{X}$ into the liquid during growth generated a concentration gradient that caused a large-scale (compared to $\lambda$ ) Mullins-Sekerka-like instability of the $\alpha \beta$ growth front at values of $V$ larger than a threshold value $V_{c}[21,22]$. Spiral two-phase dendrites were observed at $V>>V_{c}$ and appeared in the form of isolated objects. Their tip region was thus growing under nearly free-growth (virtually zero $G / V$ and infinite primary spacing) conditions [23-25]. The theory presented herein should therefore be applicable to them. Their growth direction, like those of doublons and two-phase fingers, was not fixed, but history dependent, meaning that different $s p$ dendrites had different values of their tip growth rate $v$ at fixed $V$. The explored $v$-range was $0.1-1 \mu \mathrm{ms}^{-1}$. The tip radius $\rho$ of the $s p$ dendrites was measured by fitting a parabola to the contour of the images. The extension of the tip region was between 5 and $10 \rho$. The spacing $\lambda$ was determined as the product of $v$ 
and the period of rotation of the spiral pattern. The measured $\lambda$ values turned out to be within $10 \%$ of the $\lambda_{J H}$ ones for the AB binary alloy $\left(\lambda_{J H}^{2} V \approx 10.2 \mu \mathrm{m}^{3} \mathrm{~s}^{-1}\right)$ over the whole experimental $v$ range. The $\rho$ values showed a similar variation, with $\rho \approx 0.75 \lambda_{J H}$ in average. Most importantly, two $s p$ dendrites growing simultaneously, side by side, had the same values of $\lambda, \rho$ and tip temperature. There is thus a clear indication of a strong selection of both the tip radius and the eutectic spacing of $s p$ dendrites according to a $\lambda \sim \rho \sim v^{-1 / 2}$ law.

We now come to the theory of two-phase dendrite growth. A letter $c$ will designate a concentration of B, a letter $x$ a concentration of $X$ and a pair $(c, x)$ a composition of the alloy. Concentrations in the solid phases will be tagged by a subscript $s$ and average concentrations along the growth front by a bar. As a first step, we consider the long-range concentration field of a $s p$ dendrite. For ease of exposition, we assume that there is no cross-diffusion in the liquid and that the diffusion coefficients of $\mathrm{B}$ and $\mathrm{X}$, called $D$ and $D_{x}$, respectively, have the same order of magnitude, as is in fact often the case in the experiments. Then an Ivantsov equation holds for each component separately: we have

$$
\frac{v \rho}{D}=F^{I v}\left(\Delta_{c}\right), \frac{v \rho}{D_{x}}=F^{I v}\left(\Delta_{x}\right),
$$

where

$$
\Delta_{c} \sim \frac{\bar{c}-c_{\infty}}{\bar{c}-\bar{c}_{s}} ; \Delta_{x} \sim \frac{\bar{x}-x_{\infty}}{\bar{x}-\bar{x}_{s}}
$$

The average concentration $\bar{x}$ is defined by the equation

$$
\bar{x}=\eta x^{\beta}+(1-\eta) x^{\alpha},
$$

where $\eta$ is unknown. Similar equations hold for $\bar{c}, \bar{c}_{s}$ and $\bar{x}_{s}$. By elimination of $v \rho$, Eqs. (7) lead to a relationship between $\Delta_{c}$ and $\Delta_{x}$, and thus between the average concentrations. Knowing that $D_{x} \approx D$, we rewrite Eqs. (7) in the form

$$
\Delta_{c} \sim \Delta_{x}
$$

and

$$
\frac{v \rho}{D} \sim \frac{\Delta_{x}}{\ln \left(\Delta_{x}^{-1}\right)}
$$

Regarding the short-range part of the concentration field, we approximate the spiral substructure near the dendrite tip by some effective lamellar structure. The resulting uncertainty in the definition of $\lambda$ and $v$ is within the limits of the scaling theory proposed here. Then, according to Eqs. (3) and (5), and assuming that $\lambda / \lambda_{J H}$ is kept constant at a value close to 1 in agreement with the experimental measurements:

$$
P \frac{\lambda v}{D} \sim \Delta_{E}
$$

and

$$
\frac{d_{0}}{\lambda} \sim \Delta_{E}
$$

where $\Delta_{E}$ is given by Eqs. (4) and (6). There are apparently twelve unknown concentration variables in Eqs. (10) to (13), but their actual number is only two due to the four relationships defining the average concentrations and the six relationships giving, for each phase, three equilibrium concentrations as a function of the fourth one. Thus, at this stage there are six unknowns, say, $x^{\alpha}$, $x^{\beta}, \eta, v, \rho$ and $\lambda$, at fixed $\left(c_{\infty}, x_{\infty}\right)$, and four relationships between them.

We now look for additional relationships. Figure 2 schematically represents the isothermal section of the ABX phase diagram at the temperature $T$. It features: two two-phase domains delimited by the intersections of the liquidus and solidus surfaces by the $T$-plane; the point of intersection $\left(c_{u}, x_{u}\right)$ of the univariant groove with the $T$-plane; the points $\left(c_{s u}^{\alpha}, x_{s u}^{\alpha}\right)$ and $\left(c_{s u}^{\beta}, x_{s u}^{\beta}\right)$ on the solidus lines that are in equilibrium with $\left(c_{u}, x_{u}\right)$; the triangular three-phase domain delimited by the conjugation lines between, $\left(c_{u}, x_{u}\right),\left(c_{s u}^{\alpha}, x_{s u}^{\alpha}\right)$ and $\left(c_{s u}^{\beta}, x_{s u}^{\beta}\right)$.

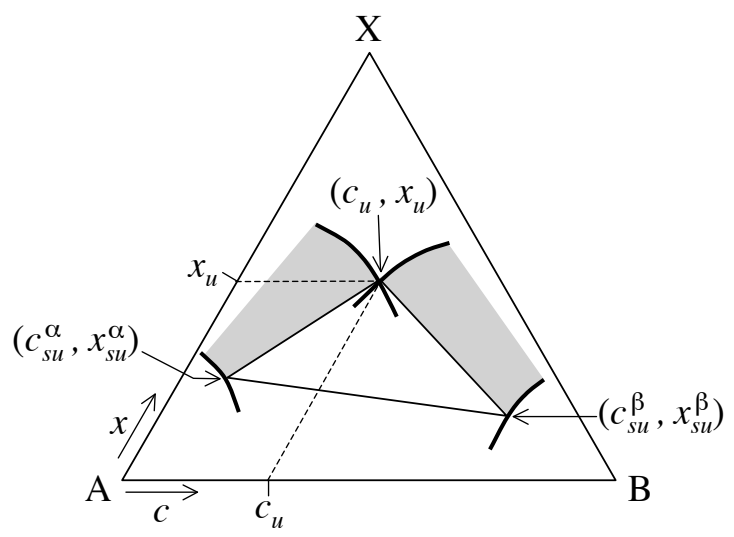

FIG. 2: Isothermal section of the phase diagram of the ABX alloy in Gibbs' triangular coordinates. Grey areas: two-phase domains. Triangle: three-phase domain.

For the sake of clarity, we begin by considering the particular case of a system having A $\leftrightarrow$ B symmetry (Fig. $3 \mathrm{a})$. In other words, we assume that the isothermal section of the phase diagram is symmetrical with respect to the $x=1-2 c$ axis and that $\left(c_{\infty}, x_{\infty}\right)$ is on this axis. Incidentally, these conditions were approximately fulfilled in the experimental system in which $s p$ dendrites were observed. Under these conditions, the $s p$ dendrites must satisfy the symmetry requirements $\eta=1 / 2$ and $x^{\alpha}=x^{\beta}$. The number of unknowns is now four, say, $\bar{x}, v, \rho, \lambda$. On the other hand, the $\mathrm{A} \leftrightarrow \mathrm{B}$ symmetry also imposes that $\bar{x}=1-2 \bar{c}$ and $\bar{x}_{s}=1-2 \bar{c}_{s}$, so that Eq. (10) becomes an identity and is no longer part of the equations of the problem. One additional relationship arising from the interaction between the long-range and the short-range 
dynamics must be found. The experiments clearly suggest that there is a linear, or almost linear, relationship between $\lambda$ and $\rho$. We therefore write:

$$
\lambda \sim \rho .
$$

Given that $\lambda \sim \lambda_{J H}$, Eq. (14) also implies that $\lambda \sim \rho \sim$ $v^{-1 / 2}$. Presumably, Eq. (14) arises from the fact that $s p$ dendrites cannot grow with $\rho<<\lambda$ for geometrical reasons while, on the other hand, $s p$ dendrites with $\rho>>$ $\lambda$ are unstable with respect to a decrease in $\rho$ that would make them grow faster. So, the only $\rho$ vs $\lambda$-range in which $s p$ dendrites can stabilize is the one given by Eq. (14).

From Eqs. (11), (12) and (14) one finally obtains

$$
\Delta_{E} \sim \frac{P \Delta_{x}}{\ln \left(\Delta_{x}^{-1}\right)}
$$

Both the quantities $P$ and $1 / \ln \left(\Delta_{x}^{-1}\right)$ are substantially smaller than unity. Thus $\Delta_{E} / \Delta_{x}<<1$ and $x_{u}-\bar{x}<<1$. We can therefore approximate $\Delta_{x}$ as

$$
\Delta_{x} \approx \frac{x_{u}-x_{\infty}}{x_{u}-x_{s u}} .
$$

Plugging this relationship into (15), it can be seen that $\Delta_{x}$ and $\Delta_{E}$, i.e. the driving forces for growth, now depend only on control and material parameters. Expending the equilibrium equations to the first order in $x-x_{u}$ near the univariant points, one obtains from Eq. (8)

$$
x_{u}-\bar{x} \sim \frac{c_{s u}^{\beta}-c_{s u}^{\alpha}}{2\left|\partial c^{\alpha} / \partial x_{u}\right|-1} \Delta_{E},
$$

which yields $\bar{x}$ as a function of the control and material parameters. Then one gets from Eqs. (12) (13) and (14)

$$
v \sim \frac{D}{P d_{0}} \Delta_{E}^{2}
$$

and

$$
\lambda \sim \rho \sim \frac{d_{0}}{\Delta_{E}}
$$

which completely solves the selection problem. As a last remark, we put Eq. (17) into the form

$$
x_{u}-\bar{x} \sim K\left(x_{u}-x_{\infty}\right) .
$$

It can be seen from Eqs. (15), (16) and (17) that $K$ is the product of $P / \ln \left(\Delta_{x}^{-1}\right)$ and some material parameters that generally are on the order of unity. Thus, in general, $K<<1$ and $\bar{x}$ is much closer to $x_{u}$ than $x_{\infty}$.

In the case of systems without any particular symmetry, there are two more independent unknowns (say, $\eta$ and $x^{\alpha}-x^{\beta}$ ) and one more relationship than in symmetrical systems. One additional relationship is thus required for determining all the unknowns uniquely. However, this relationship is about the details of the short-range field
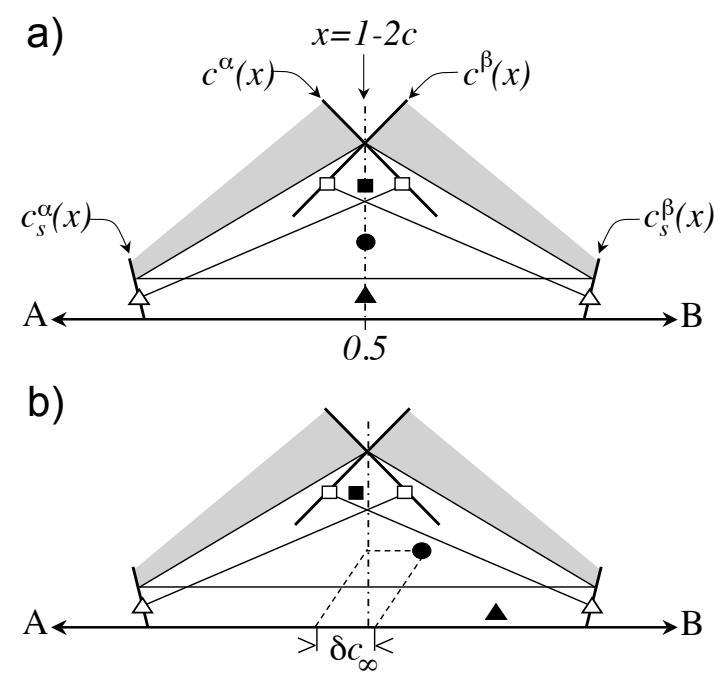

FIG. 3: Isothermal section of a phase diagram having $A \leftrightarrow B$ symmetry. a) Symmetrical system. b) Symmetry-broken system. Closed circles: $\left(c_{\infty}, x_{\infty}\right)$. Closed squares: $(\bar{c}, \bar{x})$. Closed triangles: $\left(\bar{c}_{s}, \bar{x}_{s}\right)$. Open squares: $\left(c^{\alpha}, x^{\alpha}\right)$ and $\left(c^{\beta}, x^{\beta}\right)$. Open triangles: $\left(c_{s}^{\alpha}, x_{s}^{\alpha}\right)$ and $\left(c_{s}^{\beta}, x_{s}^{\beta}\right)$.

and is of little consequence as regards the domain of existence of two-phase dendrites. As an exploratory suggestion, we therefore set $x^{\alpha}-x^{\beta}$ to zero. Keeping the same symmetrical phase diagram as above, we introduce a small departure $\delta c_{\infty}$ of the alloy concentration from the axis of symmetry and calculate the departures of $\eta, \bar{c}$ and $\bar{c}_{s}$ from their values at $\delta c_{\infty}=0$. Expending Eqs. (8) and (10) to first order and using the fact that $\Delta_{E}<<\Delta_{x}$, one gets $\delta \bar{c} \sim-\delta c_{\infty} \Delta_{E} / \Delta_{x}, \delta \bar{c}_{s} \sim \delta c_{\infty} / \Delta_{x}$ and

$$
\delta \eta \sim \frac{\delta c_{\infty}}{\left(c_{s u}^{\beta}-c_{s u}^{\alpha}\right) \Delta_{x}} .
$$

It is interesting to note that $\delta \eta, \delta \bar{c}_{s}$ and $\delta c_{\infty}$ have the same sign and that $\left|\delta \bar{c}_{s}\right|$ is substantially larger than $\left|\delta c_{\infty}\right|$. When, for instance, $\delta c_{\infty}$ is positive, the dendrite is, on average, more enriched in B than the liquid. The concentration range of existence of $s p$ dendrites, as determined by Eq. (21) and the condition $|\delta \eta|<0.5$, is delimited by the conjugation lines $\left(c^{\sigma}, x^{\sigma}\right)-\left(c_{s}^{\sigma}, x_{s}^{\sigma}\right)$. Thus, as $\bar{x}$ is close to $x_{u}$, the range of existence of $s p$ dendrites roughly coincides with the three phase domain (Fig. 3b). The operating point of the dendrite tip remains close to the univariant groove of the phase diagram as the alloy composition goes through this domain.

In conclusion, we have obtained a complete solution of the selection problem for two-phase dendrites on the condition of postulating a relationship linking the tip radius of the dendrite to the interphase spacing of the eutectic microstructure. This relationship reads $\rho \sim \lambda$ and is based on the geometry of the source of the eutectic patterns located at the tip of the dendrite. This very natural length scale selection by the underlying eutectic structure replaces the much more delicate surface tension 
anisotropy mechanism that takes place in one-phase dendrites (the so-called microscopic solvability criterion). No particular condition appears to be required for the alloy to display two-phase dendrites beyond the fact that its composition is close to a univariant groove of the phase diagram and that it solidifies in a nonfaceted way. This suggests that $s p$ dendrites should be of common occurrence in univariantly solidified ternary eutectics.

\section{Acknowledgments}

We thank M. Plapp and G. Boussinot for fruitful discussions. EAB benefited from an invited-professor position at UPMC.
[1] U. Hecht, L. Granasy, T. Pusztai , B. Böttger, M. Apel, V. Witusiewicz, L. Ratke, J. De Wilde, L. Froyen, D. Camel, B. Drevet, G. Faivre, S.G. Fries, B. Legendre, S. Rex, Mater. Sci. Eng., R Rep. 46, 1 (2004).

[2] M. D. Rinaldi, R. M. Sharp, and M. C. Flemings, Metall. Trans. 3, 3139 (1972).

[3] S. Akamatsu, M. Perrut, S. Bottin-Rousseau, G. Faivre, Phys. Rev. Lett. 104, 056101 (2010).

[4] L. Sturz, V.T. Witusiewicz, U. Hecht, S. Rex, J. Cryst. Growth 270, 273 (2004).

[5] T. Pusztai, L. Rátkai, A.Szállás and L. Gránásy, Phys. Rev. E 87, 032401 (2013).

[6] G.P. Ivantsov , Dokl. Akad. Nauk. SSSR 58, 567 (1947).

[7] M. Ben Amar and Y. Pomeau, Europhys. Lett. 6, 609 (1988). See also: D. Kessler, J. Koplik and H. Levine, Adv. Phys. 37, 255 (1988).

[8] E. A. Brener, J. Cryst. Growth 99, 165 (1990).

[9] E. A. Brener and V.I. Mel'nikov, Adv. Phys. 40, 5397 (1991).

[10] Y. Pomeau and M. Ben Amar in Solids far from equilibrium, edited by C. Godrèche, (Cambridge University Press, Cambridge, 1992), p. 365.

[11] M. Ben Amar, E.A. Brener, Phys. Rev. Lett. 71, 589 (1993).

[12] E. A. Brener, Phys. Rev. Lett. 71, 3653 (1993).
[13] M. Ben Amar, E.A. Brener, Phys. Rev. Lett. 75, 561 (1995).

[14] S. Akamatsu, G. Faivre, T. Ihle, Phys. Rev. E 51, 4751 (1995).

[15] S. Akamatsu, G. Faivre, Phys. Rev. E 61, 3757 (2000).

[16] G. Boussinot, C. Hüter, E. A. Brener, Phys. Rev. E 83, 020601(R) (2011).

[17] K.A. Jackson and J.D. Hunt, Trans. AIME 236, 1129 (1966) and references therein.

[18] A. Karma and A. Sarkissian, Metall. Mater. Trans. 27A, 635 (1996).

[19] M. Ginibre, S. Akamatsu and G. Faivre, Phys. Rev. E 56, 780 (1997).

[20] S. Akamatsu, M. Plapp, G. Faivre and A. Karma, Phys. Rev. E 66, 030501 (R-4p.) (2002).

[21] W.W. Mullins and R.F. Sekerka, J. Appl. Phys 35, 444 (1964).

[22] M. Plapp, A. Karma, Phys. Rev. E 60, 6865 (1999) and Phys. Rev. E 66, 061608 (2002).

[23] Y. Saito, C. Misbah, H. Müller-Krumbhaar, Phys. Rev. Lett. 63, 2377 (1989)

[24] S. Akamatsu and T. Ihle, Phys. Rev. E 56, 4479 (1997).

[25] S. Gurevich, A. Karma, M. Plapp, R. Trivedi, Phys. Rev. E 81, 011603 (2010). 